\title{
La réception de la littérature en Acadie : sur quelques débats autour de la critique dans L'Évangéline et L'Acadie Nouvelle
}

\section{David Bélanger, Université Laval}

L'analyse des controverses et des querelles suppose divers supports méthodologiques, lesquels, selon les visées des analystes, sociologues, historiens ou littéraires, apportent des réponses différentes. Du coup, un projet tel que celui d'analyser l'évolution des querelles et controverses littéraires en Acadie entre 1962 et 2010, et, de surcroît, de limiter l'étude de ces querelles aux chroniques, articles et opinions du lecteur parus dans L'Évangeline puis dans L'Acadie nouvelle demande, en plus d'être situé dans son cadre spatio-temporel, à être cadré dans une optique particulière qui mènera nécessairement à des réponses particulières. Une analyse bourdieusienne permettrait de voir, par exemple, l'évolution de la légitimité et de la consécration d'auteurs tels que Ronald Després, Gérald Leblanc ou Herménégilde Chiasson dans les controverses les mettant en scène ; un historien trouverait certes à relier des mouvements sociaux tels l'avènement d'une modernité et d'une autonomie en Acadie aux discours sur la littérature publiés dans les journaux acadiens. Or, si j'ai le projet d'analyser ces controverses pour elles-mêmes, sans tenter de relier les différents discours aux actions sociales, aux réalités de champs, de légitimité ou même de qualité ; si, enfin, je désire simplement suivre les discussions et tenter de mesurer les outils rhétoriques et les perceptions à l'œuvre, je dois adopter un angle moins systémique qui aura le souci, tout pragmatique, de suivre les querelles de leur début à leur dénouement. Cette démarche, il me semble, répond bien à cette mise en garde de Gisèle Sapiro vis-à-vis d'une conception totalisatrice de la sociologie littéraire :

[L]e monde des lettres est un univers aux frontières floues et poreuses, où les conditions d'accès ne sont pas codifiées, et où aucune institution ne peut prétendre détenir le monopole du pouvoir de définir les règles du jeu [...]. Le monde des lettres est, en effet, structuré en micro-milieux [...] et en réseaux de relations informelles qui prennent souvent une forme personnalisée. (46) 
Cette «forme personnalisée » dont parle Sapiro, qui refuse qu'on l'aborde en tant que totalité fermée - à l'instar d'une œuvre littéraire, par exemple -, [r]appelle la Sociologie de l'Acteur-réseau développée par Michel Callon dans son article «Éléments pour une sociologie de la traduction ». Cet article me servira d'assise méthodologique pour la présente étude, ouvrant tantôt la porte à des concepts que je définirai plus loin, tantôt à des questions qui guideront ma réflexion. Ainsi, je tenterai de faire mienne cette aspiration de Callon : «Au lieu d'imposer [aux acteurs des controverses] une grille d'analyse préétablie, [l'analyste] les suit pour repérer comment ils définissent et associent, parfois en les négociant, les différents éléments dont ils composent leur monde, qu'il soit social ou naturel. »(177) Muni d'une méthodologie, il me faut maintenant trouver une problématisation, car, si Callon propose d'observer une controverse pour elle-même et d'en tracer la trajectoire, mon but est tout autre: je désire observer différentes controverses, en tâchant de comprendre les réseaux de chacune desdites controverses, et de proposer, sous la forme d'une synthèse, un portrait de l'évolution des positions autour de questions circonscrites à l'avance. Il faut donc déjà poser la question qui me servira à interroger les querelles ciblées. Je l'articule ainsi : Comment la problématique régionale et la qualité littéraire sont-elles traitées dans les différentes controverses, et quelle place occupent-elles ? J'aborderai, plus précisément, trois controverses : une dans les années 1960, opposant l'œuvre de Ronald Després à l'analyse d'Euclide Daigle, puis une querelle dans les années 1970 sur la réception du Djibou de Laval Goupil. Je terminerai, sous la forme d'une synthèse, avec la toute récente querelle provoquée par la publication d'un numéro spécial de la revue Nuit Blanche sur la littérature acadienne.

La première querelle revêt une importance particulière due d'abord au nombre de réactions et d'impliqués dans le débat, mais aussi à la cristallisation initiale des positions. La situation historico-littéraire joue sur l'attitude des forces en place ; Manon Laparra décèle dans cette «fin des années 1950 », un pivot ou, pour reprendre son expression, « une crise majeure des assises institutionnelles »:

Ce bouleversement des vêtures imaginaires coïncide, sans surprise, avec une crise majeure des assises institutionnelles de la société acadienne à la fin des années [19]50 et se manifeste de manière éclatante par le truchement des voix littéraires à partir de 1958, année des deux premières 
publications acadiennes modernes: Pointe-aux-Coques d'Antonine Maillet et Silence à nourrir de sang du poète Ronald Després. Ces ouvrages et tous ceux qui vont suivre participent d'un même élan, [...], celui d'une démythification de l'Acadie, ou plus exactement celui d'un renouvellement des structures mythiques. (156-57)

Crise, bouleversement et avènement de la modernité semblent se rencontrer à cette époque charnière sur laquelle je propose de me pencher, ici, par le truchement des querelles. Sans pour autant remettre en question le concept de modernité qu'utilise Laparra, il conviendra, dans mon observation de cette controverse, d'établir des réseaux en regard de ces bouleversements de l'imaginaire.

Cette querelle entre Daigle et Després débute en janvier 1963 et se termine en février de la même année. Nous pouvons la résumer en deux temps : d'abord, la prise de position de Daigle contre l'œuvre de Després, une position qui est alors condamnée quasi unanimement par plusieurs lecteurs qui défendent Després et sa poésie. Ensuite est publiée la réplique de Després lui-même qui ironise sur l'attaque de Daigle, laquelle réplique est suivie par une vive contestation et des appuis, cette fois, pour Daigle ainsi que des condamnations diverses des propos de Després, notamment pour son arrogance. Ici entre en jeu la méthode présentée par Callon. Une telle controverse, mentionne-t-il, demande qu'on s'interroge sur chacune des interventions afin de produire un portrait global des réseaux en place. Ce questionnement s'effectue sur trois plans: la problématisation (ou comment un acteur se veut-il indispensable par son intervention ? [181]), ensuite l'entre-définition des acteurs (181) (qui consiste à observer comment un acteur définit l'opposant, mais aussi comment, par opposition, il se définit lui-même), puis, finalement, la solution ou l'utopie sous-jacente du discours de chaque intervenant. Évidemment, d'autres questions méthodologiques sont posées par Callon, mais nous en resterons à celles-ci qui permettront le mieux, c'est-à-dire le plus efficacement possible, de recueillir des réponses en lien avec notre grande question initiale, laquelle, je le rappelle, porte sur la place du régional et de la qualité littéraire accordée dans les différents discours.

D'abord, Daigle marque sa désapprobation et son rejet de l'œuvre de Després en raison des dommages que celle-ci pourrait causer sur la lecture en français en Acadie. Ainsi, dit-il, «J'exprime [mon point de vue] sans passion, tout simplement parce que je 
redoute l'effet de ce livre sur la popularité de la lecture française dans notre milieu ». On a ici un bel exemple de problématisation : Daigle explique son intervention par un souci de survie du fait français - et d'une lecture en français - dans son milieu, de telle sorte que ses attaques à l'endroit de l'œuvre de Després prennent l'apparence, de façon rhétorique, d'une défense de la langue française. Daigle poursuivra ses jugements sur l'écriture de Després ; ils se résument par une référence au conte «Les habits neufs de l'empereur » de Hans Christian Andersen dans lequel, on le sait, un roi dit avoir de beaux habits alors qu'il est nu ; les sujets s'extasient devant la beauté de l'étoffe alors qu'un enfant pointe l'empereur et révèle sa nudité. Daigle s'arroge d'office le rôle de l'enfant ; toute sa présentation va en ce sens : il se définit comme le lucide, celui qui voit la réalité que certains ne savent pas voir. Il agit en cela, pour reprendre une proposition de Jacques Dubois, à l'instar des «instances sociales [...][qui] prétendent n'avoir que faire de cette 'obscurité', de ce brouillage de sens [d'une certaine littérature] » (Institution, 112), et il « se plaît à retirer prestige et assurance à la 'consommation ostentatoire' [de l'art][.] » (113) Son incompréhension des poèmes de Després devient un jugement sur le caractère universellement incompréhensible de ce type d'œuvre. Plus encore, il souligne que ce caractère «obscur» de l'œuvre de Després nuit à la communauté. Il souligne que l'esthétisme propre à cette pratique - pratique que Daigle se garde bien de relier à une pratique plus large qu'on pourrait nommer, comme Laparra, la modernité - n'a aucune valeur, sinon celle de certains individus qui feignent d'y voir quelque intérêt. Le réseau de Daigle, ainsi, est l'ensemble des lecteurs qui n'y comprennent rien, qui ne font pas partie de cette « élite » intellectuelle consacrant institutionnellement la poésie de Després. Cette institution littéraire, Daigle l'accuse donc de tartufferie : ils adulent Després sans rien comprendre à sa poésie : «Les gens peuvent continuer à dire que c'est beau et bon, même s'ils n'y comprennent rien. C'est leur affaire ». La question régionale est centrale dans son propos : il faut que les gens du milieu lisent; il ne faut pas que la supposée qualité littéraire - lire, ici, celle reconnue par l'institution - les freine dans leur lecture. Les répliques sont nombreuses. Thérèse Châtillon, Vincent Joly, Roméo Savoie et JeanClaude Cardinal, notamment, se prononcent sur cette critique. En général, la problématisation s'articule sur un point : la critique de Daigle est illégitime, il n'a pas le droit de citer sur de tels sujets ; ses connaissances littéraires sont insuffisantes. Pour 
encore reprendre les réflexions de Dubois, on assiste, devant l'émergence d'une poétique moderne en Acadie, à l'apparition de nouveaux codes que Daigle ne maîtrise pas. Du coup, il devient un paria :

C'est véritablement au fonctionnement d'un code de reconnaissance et de sélection que nous avons affaire, et ce code est à double office : en même temps que les membres du groupe sélectionnent les éléments du corpus littéraire [...], ces œuvres élues distinguent les membres du groupe; ne pas adhérer instantanément au code, c'est s'exclure du groupe, devenir un paria. (Institution, 116-17)

Roméo Savoie exprime mieux que tout autre cette nouvelle définition de la littérature, celle-là même qui ovationne le travail de Després. Cette définition est résolument tournée vers des codes formels et esthétiques plus hermétiques : "Que sert à une lecture d'être populaire si elle est toute cuite, toute mâchée et toute digérée ? » Son réquisitoire, en faveur d'un art pour l'art, vise à montrer à la fois que Daigle ne comprend rien aux canons littéraires modernes (il écrit : «La compréhension d'une chose ne fait pas sa beauté ! [...] Elle est belle en soi, par sa nature ») et que l'opinion telle qu'elle est exprimée par Daigle - comme elle serait exprimée par quiconque, peut-on comprendre ne peut être considérée avec intérêt tant que cette opinion n'est pas formulée selon le bon entendement du canon littéraire. Cela s'approche de ce qu'affirme Terry Eagleton dans son ouvrage Critique et théorie littéraire :

Être diplômé d'État en études littéraires signifie être capable de parler et d'écrire d'une certaine façon. [...] Personne ne se souciera de ce que vous direz ni des positions modérées, radicales, conservatrices ou extrémistes que vous adopterez pourvu qu'elles soient compatibles avec une forme spécifique de discours [...]. Les études littéraires, en d'autres mots, sont une question de signifiants et non de signifiés. (197-98)

Il faut, en définitive, maîtriser un certain habitus culturel pour disposer de la « compétence et notamment sous la forme d'un code [...] [qui] permet d'établir des distinctions, de reconnaître les œuvres et de tenir sur les produits de l'art un propos structuré ». (Dubois, Institution, 180-1) C'est en ce sens que l'on peut comprendre la réplique des opposants à Daigle : ils définissent les lecteurs qui ne saisissent pas la pertinence de la poésie de Després comme des fautifs ; ces derniers doivent s'ajuster à un canon qui les dépasse largement, et leur nombre - c'est-à-dire la popularité d'une œuvre - n'a aucune importance puisque la littérature dépasse ses lecteurs. Du coup, les partisans 
de Després se définissent comme initiés, modernes - ils s'inscrivent dans un vaste réseau qu'est le canon littéraire, avec Arthur Rimbaud, Charles Baudelaire, Pierre Jean Jouve, Jules Supervielle, Blaise Cendrars, Hector de Saint-Denys Garneau, et ils citent de surcroît l'institution québécoise qui a décerné un prix à Després. Ils mesurent leur expertise à l'aune de ce que Pascale Casanova nomme le «méridien de Greenwich littéraire $^{1} \gg$. La valeur littéraire occupe donc toute la place dans leur propos, et ils soulignent que Després est un des rares avatars de la qualité littéraire en Acadie. Cette valeur ne saurait cependant cohabiter avec une notion de lecture régionale : la qualité précède le lectorat, le transcende, et s'y arrêter consisterait à rabaisser l'exigence de la littérature.

Després ajoute peu au débat, sinon en radicalisant certains propos. Ainsi, il parle de la querelle de l'Ancien contre les modernes, passant au registre agonique pour définir Daigle : Daigle n'est pas apte à mener un débat ; Després marque bien que la discussion avec Daigle ne vaut pas la peine, et il isole le critique, il en fait un dissident. Després inscrit sa propre poésie dans une réception consensuelle : sa qualité ne peut être remise en cause, remarque-t-il, et celui qui l'ose est seul - l'Ancien - mal adapté au monde moderne. Il fallait citer cette implication de Després dans le débat pour saisir l'importance de la réaction qui suivra. Deux problématisations se concurrencent dans cette réaction : la première veut défendre le droit du lecteur à son opinion, la seconde marque une volonté, paradoxale, de ne pas confondre qualité littéraire et chauvinisme régional. C'est Jean Lessard qui écrit : «Devons-nous usurper de la commune nationalité d'un auteur et proclamer ses œuvres tout simplement pour en illustrer sa terre d'origine ? » Lessard marque ici l'existence d'une littérature acadienne au-delà de Després et des canons littéraires modernes que son œuvre suppose. Les nouveaux intervenants reprennent les définitions de Daigle, mais accentuent la condamnation d'une certaine élite - on parle de «docte-secte», de dénigreurs et de prétentieux. La valeur littéraire devient ainsi l'objet d'une mafia, d'une cour, d'une religion avec sa propre cabale. La réaction en faveur du lecteur appelle aussi une littérature plus populaire et régionale ; Lessard explicite cette volonté en marquant l'existence d'une littérature particulière à l'Acadie non-centrée sur les canons littéraires inaccessibles aux lecteurs dont Després est devenu l'icône, non-centrée sur cette seule inaccessibilité, sur la maîtrise 
particulière d'un code. Ainsi, un certain Antonin, partisan de Daigle, écrit: "C'est le pauvre artiste qui se fait pousser la barbe pour se faire identifier comme artiste ; c'est aussi le pauvre intellectuel qui dégueule son vocabulaire pour se faire croire mieux équilibré que les autres ». La question de la modernité semble inhérente à cette querelle, mais non centrale : en fait, prédomine dans le débat, on l'a vu, cette opposition entre spécialiste et lecteur commun. Si le spécialiste est attaché à un art moderne (Vincent Joly attaque Daigle en l'accusant de n'avoir rien lu depuis Victor Hugo), les partisans du lecteur commun ne s'opposent pas à cette modernité : ils s'opposent à l'hermétisme. Une triple adéquation peut être cependant décelée, basée sur la géographie (le régionalisme contre l'universalisme), la valeur (l'hermétisme contre l'accessibilité) et le temps (la modernité contre l'ordre ancien). Il va sans dire que les partisans de Després, s'ils défendent leur attachement à une pratique mondiale et universelle de la littérature (lire ici, qui se rapproche du «méridien de Greenwich littéraire » dont parle Casanova), ils défendent en même temps un hermétisme, celui d'un groupe d'intellectuels au fait de cette «évolution » des canons et pouvant en comprendre la portée. Ces deux valeurs se lient sous le thème de la modernité : être moderne exclut, d'une certaine manière, l'attachement spatio-temporel au profit d'un attachement à l'Autre, à la Littérature. Il s'agit bien d'une tendance assimilatrice : «L'assimilation [...] est le 'degré zéro' de la révolte littéraire, c'est-à-dire l'itinéraire obligé de tout apprenti écrivain venu d'une région démunie politiquement et/ou littérairement lorsqu'il n'a à sa disposition aucune ressource littéraire et nationale » (297). Alors qu'apparaissent des œuvres nouvelles, des cours universitaires et, sous peu, une maison d'édition, il est normal que les réseaux en place tentent d'installer leur vision utopique de la littérature. Cette querelle doit être maintenant comparée à une controverse dont le sujet sera une œuvre populaire, nous pourrons ainsi mieux cerner l'évolution des réseaux ; cela est d'autant plus intéressant que la querelle du Djibou survient dans une période de construction des institutions, construction qui, je le rappelle, remplace la fragilité des années 1960.

La querelle du Djibou de 1975 s'inscrit dans une plus grande controverse qui débute, elle, en 1974 et qui survient après le refus de L'Évangeline de publier des lettres écrites en chiac. Les acteurs et les entre-définitions se ressemblent beaucoup dans un cas comme dans l'autre, aussi irons-nous, pour clarifier certaines positions, chercher dans les 
propos de la querelle du chiac pour analyser celles formulées autour du Djibou, pièce de théâtre populaire de Laval Goupil. Les hostilités autour du Djibou débutent par une critique dans l'opinion du lecteur de Clarence Comeau. Ce dernier dénonce notamment l'insuffisance de l'œuvre - insuffisance artistique et intellectuelle - et ajoute, en conclusion: «Le Djibou, ce n'est pas ce qu'il dit comme ce qu'il n'a pas su dire. Par exemple, pour écrire en plus de l'Art de bien écrire [...] il faut avoir aussi de quoi à dire et de préférence les choses les plus pressentes vu la situation en Acadie ». On retrouve ici la position contraire à celle défendue par Després et l'élite littéraire : alors qu'on privilégiait l'art pour l'art, peu importe ce qu'on pouvait y comprendre ; Comeau exige du Djibou qu'il passe un message, notamment sur la condition en Acadie. Comeau, de plus, compare la pièce de Goupil à une pièce de Raymond Leblanc et remarque le peu de sérieux intellectuel de la première par rapport à la seconde. L'entre-définition est subtile, puisque Le Djibou est dénoncé pour son inaptitude à traiter pertinemment de la réalité acadienne ; or, cela ne le confine à aucun réseau particulier, sinon à celui de la médiocrité. Cependant, et c'est là qu'il faut voir ce qui précède cette querelle, les références de Comeau tressent d'elles-mêmes une sorte de réseau dans lequel la valeur régionale est primordiale. En effet, il parle d'abord d'un article de Marthe Corrivault, publié dans Le Soleil et repris dans L’Évangeline. Dans ce papier, la journaliste écrit, entre autres, que la pièce, jouée à Québec, sera «un succès qui ne serait pas dû qu'à l'exotisme». Comeau utilise cette critique méliorative pour marquer les défauts du Djibou: le fait que cette pièce soit reconnue à Québec, devient ainsi, sous la plume de Comeau, une sorte d'ingérence marquée par l'incompréhension des Québécois pour la réalité des Acadiens. Cet élément ouvre tout de même une réflexion institutionnelle qui est, on le verra, partie prenante des positions de Comeau et de ses défenseurs. En fait, on retrouve ici l'idée de marginalité, la révolte, le fantasme d'un retrait de l'ordre établi lequel est représenté, ici, par le Québec, véritable métropole francophone de laquelle il convient de s'affranchir. De plus, Comeau, par sa comparaison de l'œuvre de Goupil avec l'œuvre de Raymond Leblanc s'inscrit dans un réseau, celui d'un canon littéraire acadien, que mettent en place les éditions d'Acadie depuis leur fondation trois ans plus tôt. Le même Leblanc, dans une lettre de décembre 1974, écrit, en lien avec la «querelle du chiac » : «La poésie de Guy Arsenault, celle qui exprime l'Acadien de Moncton et 
des environs, celle qui s'exprime dans le langage d'une majorité de la population acadienne d'ici, sera-t-elle interdite dans l'Évangéline et le milieu scolaire ? et Leblanc termine sa longue lettre : "Ça serait trop vous demander que de vous prononcer $[\ldots]$ contre le système capitaliste et pour la libération du peuple acadien ? ». Ainsi, le réseau duquel se réclame Comeau est très près des valeurs régionales, et d'une certaine marginalité discursive: bien exprimer la réalité acadienne constitue, dès lors, une garantie de qualité littéraire. D'ailleurs, Comeau titrait, dans la querelle du chiac, une année auparavant: «Les Acadiens se moquent pas mal de la valeur littéraire ». Dans cette lettre à L'Évangéline, il écrit : « Pourrait-on savoir pourquoi les Acadiens, dans leur langage et articles pour préciser, n'ont pas de forme littéraire si réellement cela est indispensable ? Qui à L'Évangéline décide de la forme littéraire des Acadiens et de quels Acadiens parlez-vous? » Ce que recherche Comeau, c'est une forme adaptée à une identité - l'Acadie - et il repousse toute influence, toute ingérence, toute mainmise d'un autre canon que celui qui serait propre à l'Acadie. Ce réseau duquel il se réclame, du coup, rejette l'influence française - la querelle du chiac montre bien que la langue normative devient symbole de colonisation - et l'influence québécoise - la remarque de Comeau sur la critique du Soleil est ici révélatrice. De surcroît, en défendant la pratique moins classique de Leblanc, il défend un canon plus régional, installant sa vision de la littérature dans «un mouvement collectif de réaction contre l'emprise des discours dominants et de perturbation des formes communicationnelles les plus contrôlées » (Dubois, Institution, 217). Cette littérature régionale, nous dit Dubois, cherche donc à s'inscrire dans une marge par son rejet des codes littéraires en place. Cela, ajoute Casanova, est la « révolte » propre aux naissances des littératures :

Tous les intellectuels des «premières générations littéraires » $[\ldots]$ ont compris à la fois le phénomène de l'annexion littéraire par les espaces dominants dont ils étaient les victimes et la nécessité où ils étaient de créer une distance et une différence. [...] Seules des productions littéraires déclarées et constituées comme spécifiques et nationales peuvent permettre de mettre fin à la dépendance des écrivains à l'égard de l'espace littéraire (et politique) dominant. (314-15)

On sent ainsi le souci chez Comeau de «déclarer» comme «spécifique et nationale » la littérature acadienne ; cette exigence l'amène à un geste de condamnation (Laval Goupil) et a un geste de célébration (Raymond Leblanc). 
Denis Sonier, qui deviendra éditeur aux Éditions de la Francophonie, est le premier à répliquer. Cette réplique ne défend pas l'autotélisme de la littérature, comme ce fut le cas dans la querelle Daigle-Després. En fait, Sonier aborde également le thème de la représentation nationale en art pour répliquer à Comeau : «La pièce de Laval Goupil a des faiblesses mais avant de les supposer ayez la décence d'essayer de comprendre l'essentiel du message ». Une autre réplique, signée L. Noël, accentue cette idée de message incompris, en mentionnant que la pièce de Goupil montre bien l'Acadie telle qu'elle est, alors que Comeau, accuse-t-il, est un révolutionnaire qui voudrait que la pièce prêche le changement. Cette accusation de «volonté révolutionnaire » est importante, car ce semble être ce qui définit le mieux les deux réseaux : d'une part, on veut montrer l'Acadie (Goupil, Sonier), d'autre part, on veut changer les choses, opérer un mouvement. C'est l'essence des propos de Ginette Bérubé, appuyant Comeau, qui définit les « choses essentielles pour une pièce de théâtre »: «elle doit servir avant tout les personnes dont elle parle ou la communauté qu'elle veut faire parler ». Et elle ajoute, sous forme de reproche: «Le Djibou aurait pu parler de la laideur des valeurs capitalistes ». Noël Leclerc clarifie la définition du réseau de Comeau, en marquant son dédain pour la pièce de Goupil : «Une quétainerie demeure une quétainerie même si elle se rend à Windsor ou même en Europe ! Si le Djibou a du succès, c'est bien parce qu'il y a encore beaucoup de gens [...] qui se font des illusions pour maintenir le statu quo et se contentent de théâtre de salon ». La problématisation du réseau de Comeau semble claire : il faut parler de l'Acadie et officier des changements, une révolution identitaire (liée au chiac) et idéologique (qui mettrait fin au capitalisme, détruirait le statu quo). L'autre réseau ne semble pas s'offenser de la définition dont il est l'objet : il représente le statu quo, la littérature n'a pas à changer le monde, elle n'a qu'à le montrer. Gérald Leblanc, dans une lettre intitulée «L'Université sert à quoi... à qui ? », qui paraîtra quelques jours après la fin de la querelle du Djibou, écrit : «L'Université de Moncton, ça pue la piastre et le statu quo. Ça sent l'usine linguistiquement stérile. Chef de file de la population acadienne ? Dans la réalité d'un peuple enfargé dans un engrenage capitaliste avec sa parlure acadienne étranglée ». Il est intéressant de voir que l'élite littéraire des années soixante, universitaire et institutionnalisée - la docte-secte dénoncée par les défenseurs de Daigle - devient une élite financière, linguistique et, certes, intellectuelle, 
mais dont la légitimité littéraire semble fragilisée - aucun des deux réseaux ne s’y réfère, bien qu'elle semble dénoncée par Leblanc. Les universités, ainsi, n'enseigneraient pas les vrais auteurs acadiens, elles se retrancheraient derrière une idée colonisée - à la France, au Québec - du peuple acadien, et elle passerait à côté de la vraie littérature d'Acadie. S'effectue donc un glissement: le réseau défendant la littérature, celui qui semble s'inscrire dans un canon littéraire, défendre la valeur du littéraire, ce réseau reprend les positions de Daigle : la littérature doit parler pour les gens d'ici, des valeurs d'ici, elle doit s'inscrire dans notre milieu et se détacher des canons étrangers. Ainsi, la valeur littéraire, dans la querelle de 1962, s’opposait clairement à la problématique régionale : la littérature s'inscrivait dans l'art et ne devait en aucun cas - du moins ce n'était pas nécessaire - parler de l'Acadie. En 1975, l'œuvre n'a de valeur littéraire qu'en fonction de sa représentation de la société acadienne - cela est d'autant plus frappant que, de part et d'autre de la controverse, les deux réseaux ne parlent pas, en soi, d'esthétique ou de canon - du moins de façon explicite - ils parlent de message et d'Acadie. Mais une certaine réserve doit être ici énoncée: si Comeau prône, à l'instar de son réseau, une révolution, il se rattache, d'une certaine manière, à une critique en place au niveau international, soit, la critique marxiste qui «s'est toujours astreinte à inscrire l'activité esthétique dans le devenir socio historique » (Dubois, «Critique », 57). Se détachant donc, sous l'angle linguistique, de la mainmise idéologique française, Comeau et Leblanc reprennent néanmoins le fonctionnement de sa critique, formant une adéquation entre la qualité littéraire et le discours social.

En guise de conclusion, il faut ajouter que le même réseau - celui de Gérald Leblanc et Clarence Comeau, notamment - se remettra en branle lors de la réception de la Géographie de la nuit rouge de Leblanc. Nelson Landry, dans l'Acadie Nouvelle, parlera d'un «trip difficile à suivre ». Landry, rapidement, sera accusé, avec moins d'ampleur, des mêmes torts que Daigle : de ne pas comprendre la littérature, de ne pas pouvoir se prononcer sur celle-ci. La dynamique initié-néophyte reprendra rapidement ses droits, notamment dans certaines réceptions critiques controversées - pensons aux critiques dures de David Lonergan contre des œuvres publiées par Grande Marée, ou à la réception de C'est pour quand le Paradis de Claude LeBouthillier. Sans aller jusqu'à l'analyse, il apparaît néanmoins intéressant de s'attarder quelque peu à la querelle autour du magazine 
Nuit Blanche de novembre 2009, une querelle récente qui reprend, avec quelques transformations intéressantes, les définitions des réseaux précédents. Claude Lebouthilier accuse, dans sa chronique de L'Acadie nouvelle, le snobisme de l'institution littéraire visà-vis des œuvres populaires. C'est en ce sens qu'il écrit, à propos d'un article de Benoit Doyon-Gosselin mettant en question la remise du prix Antonine-Maillet Acadie-vie à Calixte Duguay : «Peut-être considère-t-on comme un défaut le fait qu[e Duguay] écrive une poésie accessible ? » De plus, LeBouthillier dénonce l'importance que le dossier accorde au chiac et le peu d'importance qu'on accorde à une poésie régionale du Nord il dénonce, au final, l'existence d'un canon littéraire de Moncton et exclusif à cette région. Sans trop nous attarder sur les répliques à cette critique, notons que les deux nouveaux réseaux créés forment une sorte de synthèse des précédents : LeBouthillier représente, à l'instar de Daigle et des opposants à Comeau, un réseau défendant la littérature acadienne populaire, dont la valeur régionale est primordiale ; il s'oppose à tout un réseau snob qui n'accorde de l'importance qu'aux œuvres inaccessibles (soit esthétiquement élaborées ou écrites en $\mathrm{chiac}^{2}$ ). Finalement, LeBouthillier fait l'éloge de l'édition acadienne dans sa richesse et sa diversité. L'autre réseau se définit plutôt comme défendeur d'une valeur littéraire (Pénélope Cormier écrit: «Doit-on s'étonner que la recherche esthétique soit au centre de la critique universitaire plutôt que l'accessibilité [?] [...] C'est le contraire qui me scandaliserait ») et l'étude d'ouvrages en chiac, plutôt que d'être justifiée par leur caractère régional, semble reposer sur le même souci de ne traiter que de qualité littéraire. La trajectoire des réseaux demeure néanmoins intéressante et ouvre la porte à diverses hypothèses - en ce qui a trait au rôle de l'Université dans les polémiques et à l'idée utopique aujourd'hui en place dans les réseaux. Ainsi, le silence de l'Université du débat littéraire dans les années soixante-dix peut marquer le désir centripète du réseau en place de se détacher de la contiguïté d'une francophonie canadienne dont parle Doyon-Gosselin : 1'Université était alors représentée en littérature par Després, mais aussi par Maillet, deux auteurs primés à l'extérieur de l'Acadie. Paradoxalement, cette volonté centripète est marquée par une production florissante et par la mise en place d'une institution solide (le prix France-Acadie en 1979, Perce-Neige à la même époque, l'Association des écrivains acadiens, etc.) Dans les années 2000, cette volonté centripète est disparue et il semble plutôt primer, dans les discours, une volonté 
de contiguïté : Nuit blanche, revue québécoise, est une représentation de cette contiguïté. Or, à cette volonté de légitimer la littérature acadienne au-delà du lectorat acadien s'ajoute un discours défaitiste sur l'effritement des institutions et le départ d'auteurs acadiens vers le milieu éditorial québécois ou franco-ontarien. François Paré projette une telle réaction : «Parmi les cultures de l'exiguïté, les minoritaires sont celles qui tendent le plus à sacraliser l'autodestruction » (28). Ce discours autodestructeur s'attache seulement, en ce qui à trait à l'Acadie, à l'institution, car la production et la voix acadienne, de leur côté, ne semblent guère perdre de leur portée.

C'est ainsi peut-être qu'on peut recevoir le dernier roman de France Daigle, écrivaine acadienne vivant à Dieppe, mais publiant à Montréal. Pour sûr, œuvre immense, chante à sa manière l'Acadie, joue sa différenciation, sans se cloîtrer cependant : les assises universelle telles que la littérature mondiale, la psychanalyse et le jeu métatextuel se retrouvent au centre du projet. Si les «petites [littératures], celles que la grandeur des unes excluait, se sont exténuées dans le morcellement et la diversité », le propre des grandes littératures, nous dit Paré, est de «s'efforce[r] de créer les conditions, hautement sacralisées, de l'universalité »(25). Le statut de l'œuvre de Daigle semble ambivalent : l'action se déroule en Acadie, la problématique acadienne et linguistique y est importante ; le roman est publié au Québec, la portée formelle et universelle y prédomine. Le régional habite du coup le littéraire, en devient le modèle de différenciation même. Bourdieu écrit :

Autrement dit, plus le champ est en mesure de fonctionner comme le lieu d'une concurrence pour la légitimité culturelle, plus la production peut et doit s'orienter vers la recherche des distinctions culturellement pertinentes dans un état donné d'un champ déterminé. (59)

Car la littérature acadienne, dans un modèle de contiguïté, atteint vraiment l'autonomie littéraire de ses champs ; la problématique acadienne devient la véritable stratégie de distinction dans une littérature-monde où se joue, à grande échelle, la grande concurrence culturelle. 


\section{Bibliographie}

Antonin. « Critique de la critique d'une critique ». L'Evangéline 12 février 1963.

Bérubé, Ginette. «Qu'avez-vous compris au djibou ? ». L'Evangéline 27 août 1975.

Bourdieu, Pierre. «Le marché des biens symboliques ». L'année sociologique 22 (1971): 49-126.

Callon, Michel. «Éléments pour une sociologie de la traduction : la domestication des coquilles Saint-Jacques et des marins-pêcheurs dans la baie de SaintBrieuc ». L’année sociologique 36 (1986) : 169-208.

Cardinal, Jean-Claude. «Une critique d'une critique ». L'Evangéline 25 janvier 1963.

Casanova, Pascale. La république mondiale des lettres. Paris : Seuil (Points), 2008 (1999).

Châtillon, Thérèse. «Cette critique signée Euclide Daigle ». L'Evangéline 18 janvier 1963.

Comeau, Clarence. «Les Acadiens se moquent pas mal de la valeur littéraire ». L'Evangéline 4 juillet 1974.

---. « Le djibou fantasque ». L'Evangéline 25 juillet 1975.

Cormier, Pénélope «Pas l'endroit pour les règlements de compte ». L'Acadie nouvelle 25 novembre 2009.

Corrivault, Marthe. «Les 'Djbiou' d'Acadie n'ont pas fini d'étonner le reste du pays ». L'Evangéline 17 juillet 1975.

Daigle, Euclide. « Une critique littéraire ». L'Evangéline 14 janvier 1963.

Després, Ronald. «Le dernier mot? ». L'Evangéline 2 février 1963.

Doyon-Gosselin, Benoit. «Les littératures en contiguïté ». Salon double [en ligne] http://salondouble.contemporain.info/antichambre/les-litteratures-en-contiguite, page consultée le 30 août 2011.

Dubois, Jacques. L'institution de la littérature. Bruxelles : Labor (coll. Espace Nord), 2005 (1978).

---. «Pour une critique littéraire sociologique». Le littéraire et le social. Éléments pour une sociologie de la littérature. Dir. Robert Escarpit. Paris : Flammarion, 1970. $55-75$. 
Eagleton, Terry. Critique et théorie littéraire : une introduction. Paris: PUP (coll. Formes sémiotiques), 1994.

Joly, Vincent. «Les poètes ne courent pas les rues ». L'Evangéline 21 janvier 1963.

Laparra, Manon. «De Memramcook en 1881 à Moncton en 2000 : esquisse d'une trajectoire de la modernité acadienne ». La modernité en Acadie. Dir. Ghislain Clermont et Janine Gallant. Moncton: Chaire d'études acadiennes (coll. Mouvange), 2005. 147-171.

Leblanc, Gérald. «L'Université sert à quoi... à qui ? ». L'Evangéline 3 septembre 1975.

Leblanc, Raymond. «Personne n'a vu le train passer ». L'Evangéline 13 décembre 1974.

Lebouthilier, Claude. «Paix sur la terre pour les hommes de bonne volonté ». L'Acadie Nouvelle 10 décembre 2009.

---. « Critique de la critique ». L'Acadie nouvelle 19 novembre 2009.

Leclerc, Noël. « Du théâtre de salon ». L'Evangéline 25 août 1975.

Lessard, Jean. «Scalpel à nourrir de sang ». L’Evangélne 12 février 1963.

Morency, Jean. «Contribuer au rayonnement de la littérature acadienne ». L'Acadie nouvelle 23 novembre 2009.

Noël, L. « Il y a du vrai dans Le djibou » L'Evangéline 12 août 1975.

Ouellet, François, dir. «La littérature acadienne débarque »[dossier]. Nuit blanche, le magazine du livre 115 (2009) : 20-52.

Paré, François. Les littératures de l'exiguïté. Ottawa: Le Nordir (coll. Bibliothèque canadienne-française), 2001 (1992).

Sapiro, Gisèle. «Réseaux, institution(s) et champ ». Les réseaux littéraires. Dir. Paul Aron et Benoît Denis, Bruxelles : Le Cri, 2006. 44-59.

Savoie, Roméo. «Réponse à une 'critique littéraire' ». L'Evangéline 21 janvier.

Sonier, Denis. «Un article de M. Joe Connaissant ». L’Evangéline 6 août 1975.

\footnotetext{
Notes

${ }^{1}$ Elle écrit : «On peut ainsi mesurer la distance au centre d'une œuvre ou d'un corpus d'œuvres, d'après leur écart temporel aux canons qui définissent, au moment précis de l'évaluation, le présent de la littérature. En ce lieu, on dira qu'une œuvre est contemporaine, qu'elle est 'dans la course ' (par opposition à 'dépassée ' [...]) selon sa proximité esthétique avec les critères de modernité $[\ldots]$ (... (135-6).

${ }^{2}$ Rappelons à ce propos le commentaire de Pierre Bourdieu sur l'institution scolaire : «Et nombre d'agressions contre l'institution scolaire témoignent que leurs auteurs reconnaissent assez la légitimité de ses verdicts pour lui reprocher de ne pas les avoir reconnus » (75).
} 